\title{
Rezension: Silvio Hänsenberger, Die zivilrechtliche Haftung für autonome Drohnen unter Einbezug von Zulassungs- und Betriebsvorschriften
}

\author{
Philip Bärtschi *
}

Drohnen werden in den kommenden Jahren im unteren Teil unseres Luftraums immer häufiger eine Rolle spielen. Die Entwicklung wird zweifellos in Richtung völlig autonomer Systeme gehen. Schadenersatzansprüche aufgrund von Fehlentscheidungen dieser autonomen Luftfahrzeuge sind allerdings weder mittels Verschuldenshaftung noch mit der Produkthaftung durchsetzbar. Ist unser Haftungsrecht also für die neue Ära unbemannter Luftfahrzeuge überhaupt gerüstet? Dieser Frage geht der Autor der hier besprochenen Dissertation nach und präsentiert Lösungsvorschläge, wie sich autonome Nano-, Mikro- und Kleindrohnen in das bestehende Rechtsgefüge einbinden lassen. Er deckt die noch bestehenden Lücken unseres Haftungssystems in Bezug auf den Betrieb autonomer Drohnen auf und zeigt anhand theoretischer Beispiele, welche Rechtsfragen sich inskünftig stellen werden und wie ihnen begegnet werden kann.

Zitiervorschlag: Philip Bärtschi, Rezension: Silvio Hänsenberger, Die zivilrechtliche Haftung für autonome Drohnen unter Einbezug von Zulassungs- und Betriebsvorschriften, in: sui-generis 2019, S. 103

URL: $\quad$ sui-generis.ch/96

DOI: $\quad$ https://doi.org/10.21257/sg.96

* RA lic. iur. Philip Bärtschi (phb@v2sky.ch / baertschi@baertschi-legal.ch) ist Mitbegründer der vasky GmbH, welche Dienstleistungen im Bereich der Zulassung komplexer Drohnenoperationen anbietet. Er ist ferner selbstständiger Rechtsanwalt und spezialisiert auf Luftfahrtrecht. 

portflüge unbemannter Luftfahrzeuge in der Schweiz zur Realität geworden. Die Post baute in Zusammenarbeit mit dem Drohnenhersteller Matternet mehrere Standorte auf, von denen aus biologische Proben zwischen Kliniken und Laboren transportiert werden. ${ }^{1}$ Seit der Zulassung dieses Projekts schreiten die Entwicklungen im Drohnenmarkt mit immer grösseren Schritten voran und bereits sind Flugsicherungsprogramme im Aufbau, die ein Nebeneinander bemannter und unbemannter Luftfahrzeuge ermöglichen sollen. Da Drohnen nicht sprachbasiert mit der Flugsicherung kommunizieren können, werden in den kommenden Jahren neue und innovative Lösungen aufgebaut werden müssen, um den Luftverkehr zwischen herkömmlichen Luftfahrzeugen und Drohnen reibungslos zu koordinieren.

Unbemannte Flüge ausserhalb des direkten Sichtbereichs (BVLOS; Beyond Visual Line of Sight) bedürfen in der Schweiz einer Einzelfallbewilligung des Bundesamts für Zivilluftfahrt (BAZL) und sind Resultat äusserst aufwändiger Bewilligungsverfahren. Gegenwärtig operieren solche Drohnen ausserhalb des Sichtbereichs lediglich semi-autonom. Dies bedeutet, dass die Fluggeräte zwar ohne menschliche Einflussnahme eine Mission durchführen können, jedoch nicht bis zu dem Grade, dass sie autonome Entscheidungen treffen müssen und können. Den Flugweg befolgen sie nach vorgegebenen und einprogrammierten Wegpunkten. Werden kurzfristig Anpassungen notwendig, bspw. wegen konfligierendem

Mitteilung der Post vom 05. Juni 2018 über den Ausbau von Standorten, von denen aus biologische Proben per Drohnen verschickt werden.
Flugverkehr oder Wettereinflüssen, bedarf es des Eingreifens eines Operateurs, der die notwendigen Entscheide trifft und den Flugweg neu programmiert oder die manuelle Steuerung übernimmt. Es ist aber absehbar, dass in nicht allzu ferner Zukunft Drohnen vollständig autonom in unserem Luftraum operieren werden, was zu einer Vielzahl völlig neuer Haftungsprobleme führen wird. Mit dieser neuen Ausgangslage befasst sich der Autor der Dissertation.

3 Die Arbeit von Hänsenberger ist im Wesentlichen in vier Hauptteile aufgebaut; Eigenschaften autonomer Drohnen (II), Haftung für Personen- und Sachschäden am Boden (III), Haftung bei Luftkollisionen und reinen Vermögensschäden (IV) und ein Regulierungsvorschlag auf $\mathrm{Ba}$ sis bestehender Normen (V). Die Dissertation konzentriert sich in erster Linie auf vollständig autonome Systeme und somit vornehmlich auf rechtliche Probleme, die sich heute in dieser Art (noch) kaum stellen. Aufgrund der Tatsache, dass solche autonomen Systeme eigenständige Entscheide treffen können und sollen, können daraus Risiken für andere Luftraumbenützer und für Personen und Gegenstände am Boden resultieren, ohne dass dafür menschliches Einwirken ursächlich ist. Verantwortlich gemacht werden könnten lediglich Algorithmen. Um auf diese neue Art von Luftraumbenützern vorbereitet zu sein, liefert die Dissertation von Hänsenberger wertvolle Vorschläge, wie den rechtlichen Problemen zu begegnen sein wird und präsentiert eine Auslegeordnung über die Haftungssituation de lege lata und de lege ferenda. 
4 Gerade dem Problem möglicher Kollisionen in der Luft mit bemannten Luftfahrzeugen - bspw. mit Grossflugzeugen im An- und Abflug auf Flughäfen - wird man sich haftungsrechtlich widmen müssen. Da Kollisionen in der Luft nicht unter die Gefährdungshaftung der Luftfahrtgesetzgebung fallen, ist gemäss den einschlägigen Bestimmungen des ausservertraglichen Haftpflichtrechts eine Sorgfaltspflichtverletzung Voraussetzung. Die Frage, wie sich eine solche bei einem autonomen System herleiten lässt, wird von Hänsenberger untersucht. Er diskutiert die von verschiedenen Autoren vorgeschlagene Kausalhaftung für autonome Systeme (aller Art) und fordert in der Folge für Schäden bei Kollisionen in der Luft die Einführung einer solchen Gefährdungshaftung in der Schweiz für autonome Nano-, Mikro und Kleindrohnen. Diese Kausalhaftung umgeht das naturgemäss vorhandene Problem der Verschuldensfrage bei autonomen Systemen, deren Flugwege nicht durch einen Menschen programmiert, sondern vielmehr durch künstliche Intelligenz selber festgelegt werden. Resultiert aus einem Entscheid der künstlichen Intelligenz eine Kollision mit einem anderen Luftfahrzeug, so würden Geschädigte bei Vorhandensein einer Kausalhaftung nicht mit den Schwierigkeiten eines Verschuldensnachweises belastet.

5 Ein weiteres Ziel der Arbeit von Hänsenberger ist zudem die Klärung der Frage der Haftung für reine Vermögensschäden auf der Erde. ${ }^{2}$ Er beleuchtet dabei insbesondere die Luftfahrt- und Produkthaftpflicht. Der Autor schlägt vor, dass
Art. 64 LFG3 derart zu ergänzen sei, dass der Halter eines sich im Fluge befindlichen unbemannten Luftfahrzeugs, das nicht durch einen Piloten gesteuert wird, auch für den weiteren Schaden haften soll, sofern dieser Schaden durch die Verletzung einer Schutznorm entstanden ist. Ein Verschulden solle dafür aber nicht vorausgesetzt werden. Für diesen weiteren Schaden hafte der Halter im Übrigen nur bis zum Betrage der Sicherstellung, zu der er von Gesetzes wegen verpflichtet ist.

6 Schliesslich widmet sich der Autor in einem eigenen Kapitel der Frage, wie Betriebsvorschriften und Luftverkehrsregeln bei einem verbreiteten Einsatz autonomer Nano-, Mikro- und Kleindrohnen ausgestaltet sein müssten. ${ }^{4}$ Dabei schlägt Hänsenberger insbesondere vor, bei der Risikoabwägung im Umfang der Zulassungs- und Betriebsvoraussetzungen statt auf das Anknüpfungskriterium des Gewichts, vielmehr auf eine eher holistische Gesamtbeurteilung des Risikos der spezifischen Operation abzustellen. $5 \mathrm{Zu}$ kurz kommt in seinen Ausführungen allerdings die Tatsache, dass eine solche Gesamtbeurteilung in der Schweiz bereits heute im Zuge des Bewilligungsverfahrens vorgenommen wird, und zwar in Form des auf europäischer Ebene entwickelten SORA-Prozesses gemäss JARUS-Richtlinien. ${ }^{6}$ Dieses «Specific Operations Risk Assessment» hat zum Zweck, eine umfassende Beurteilung der spezifischen Risiken der beantragten

$3 \longdiv { \text { Bundesgesetz über die Luftfahrt vom 21. Dezember } }$ 1948 (LFG; SR 748.0).

S. $190 \mathrm{ff}$.

S. $208 \mathrm{f}$.

JARUS guidelines on Specific Operations Risk Assessment (SORA) der Joint Autorities for Rulemaking on Unmanned Systems (JARUS). 
Operation vorzunehmen, und zwar insbesondere in Bezug auf die sog. air risks, ground risks und technical risks. Im Umfange des SORA wird dabei vor allem Risikominderungsmassnahmen Rechnung getragen, welche der Betreiber der Drohne für den Einzelfall entwickelt hat. So wirkt sich bspw. ein Fallschirm, der bei einer ausser Kontrolle geratenen Drohne automatisch ausgelöst wird, als mitigierendes Mittel auf die Gesamtbeurteilung des Risikos, und somit auf die Anforderungen, welche die Behörde für die Operation auferlegt, positiv aus. Mit einer Vielzahl weiterer technischer und operationeller Massnahmen können die Aussichten auf eine Bewilligung komplexer Missionen zu Gunsten des Antragsstellers günstig beeinflusst werden, so z.B. durch Geofencing und mehrfach abgesicherte Luftraumüberwachungssysteme.

7 Die Dissertation schliesst mit einer leserfreundlichen Übersicht über die wichtigsten Regulierungsvorschläge, die eine Gegenüberstellung de lege lata und de lege ferenda beinhaltet.

Alles in allem bietet die rund 245 Seiten umfassende Arbeit von Hänsenberger eine umfangreiche Auslegeordnung über die anwendbaren Rechtsgrundlagen für unbemannte Luftfahrzeuge unter $30 \mathrm{~kg}$, eine Darstellung der Probleme de lege lata und einen Ausblick, wohin bei der derzeitigen Entwicklungsgeschwindigkeit die Drohnenluftfahrt hinsteuert und wie dieser Entwicklung Rechnung getragen werden kann. Dabei postuliert er die Notwendigkeit einer Haftungsverschärfung für die Betreiber solcher Systeme und stellt zutreffend fest, dass die heutige haftungsrechtlichen Situation keine genügende Basis für ein gesundes Ne- beneinander von bemannter und unbemannter Luftfahrt bilden kann. Für diese Auslegeordnung und für die detaillierte Übersicht aller in Betracht fallender Anspruchsgrundlagen für Schäden in der Luft und am Boden, verdient die Dissertation von Hänsenberger Anerkennung. Sie deckt die Lücken unserer Rechtsordnung für ein im Entstehen begriffenes, neues Zeitalter der Luftfahrt auf und lässt keinen Zweifel offen, dass Drohnen in naher Zukunft ein fester Bestandteil der Luftraumbewirtschaftung bilden werden, der auch aus rechtlicher Sicht Beachtung geschenkt werden muss.

«Die zivilrechtliche Haftung für auto-
nome Drohnen unter Einbezug von Zu-
lassungs- und Betriebsvorschriften»,
Silvio Hänsenberger, Carl Grossmann
Verlag, Berlin/Bern, 2018 - ISBN: 978-
3-941159-27-3 (gedruckte Ausgabe,
Hardcover), ISBN: 978-3-941159-28-o
(e-Book, Open Access)

«Die zivilrechtliche Haftung für autonome Drohnen unter Einbezug von Zulassungs- und Betriebsvorschriften», Silvio Hänsenberger, Carl Grossmann Verlag, Berlin/Bern, 2018 - ISBN: 9783-941159-27-3 (gedruckte Ausgabe, Hardcover), ISBN: 978-3-941159-28-0 (e-Book, Open Access) 